\title{
3D Color Body Scanning for Improved Sample Fit and Accuracy in Garment Design
}

\author{
Andre WEST* and Ashley GABEL \\ NC State University College of Textiles \\ Department of Textiles and Apparel, Technology and Management, \\ Raleigh, NC, USA \\ http://dx.doi.org/10.15221/14.205
}

\begin{abstract}
For hundreds of years, muslin has been used as the basic fabric from which most garment patterns, first round samples, or prototypes, are made. In the $21^{\text {st }}$ century much of the industry has moved into CAD systems for pattern making, however, muslin remains the stand alone fabric used in pattern making for the evaluation of fit for a large part of the industry.

Muslin is known for its low cost, simple structure, and the ease for which it can be used. Unfortunately, many end-use fabrics, particularly in apparel where a wide range of fabric structures are used, are not comparable in characteristics to muslin. In fact, muslin may only be a good basis for cotton fabric woven with a plain weave structure due to the strikingly different behaviors of various yarn sizes, fiber types, and weave or knit structures (Brown, 2001). Therefore, using muslin to drape a pattern or fit a first-sample garment can actually be detrimental to the fit process, particularly in draping where the creation of the pattern depends solely on the fabric (Joseph-Armstrong, 2008). Consequently, for an improved fit, a fabric more comparable to the ultimate end use fabric might be more appropriate when used for sampling and pattern draping.
\end{abstract}

Keywords Fitting, Patternmaking, 3D Color Body Scanning

\section{Introduction}

There are a large variety of cotton muslin fabrics that are currently on the market, forming an array of weights, drapes and finishes available to the sample room. All muslins have a few things in common: they are a lightweight, loosely woven cotton and possibly the biggest determining factor, inexpensive; especially compared to the planned final fabric. Most sample rooms use whatever they have on hand and do not consider the fabric characteristic as a large part of the garment evaluation. Information is then gathered from the fit sample, decisions are made on the quality of the fit. A fabric that could better represent the finished fabric could improve the fitting and sampling process. Because there is no standard fabric currently in existence to replace muslin for fitting, there is the opportunity to develop a portfolio of muslin equivalents better suited to the sample maker's needs. In the long run, this method could lead to less design iterations.

If we look at the pattern paper that many sample makers use in Figure 1 we see the paper that is used to cut the fabric pattern piece has useful information on it; the muslin itself has none. The numbers represent the direction and help with drafting as well as vertical and horizontal alignment. With the revolution of digital fabric printing it is relatively easy to reproduce an enhanced grid pattern on the fabric in small quantities. Figure 2 represents a grid based on $1 / 8^{\text {th }}$ inch increments. Using industry pattern making software data in conjunction with Adobe Illustrator, the researchers were able, in Figure 3, to print the same sleeve pattern rendered with a grid pattern similar to or more advanced than the information found on the pattern paper with precise visual measurements.

* Tel 614.961.9121, ajwest2@ncsu.edu 


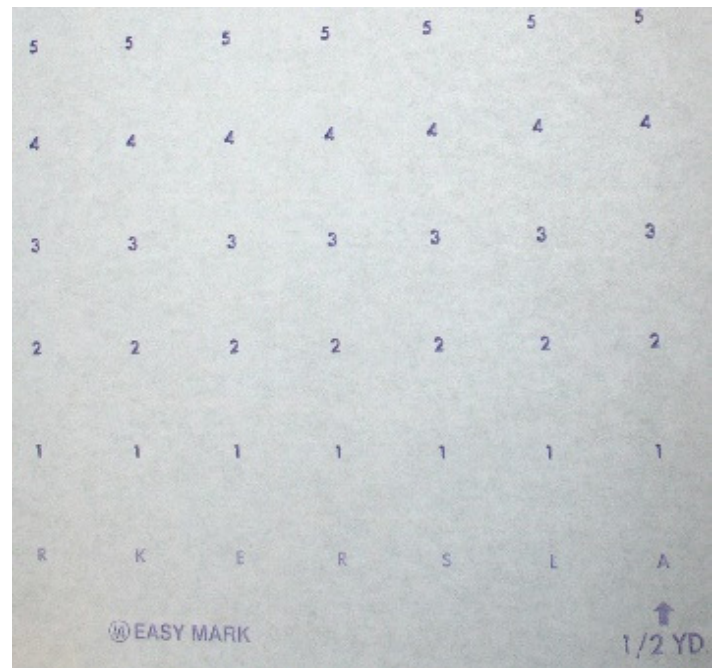

Figure 1: Pattern Paper

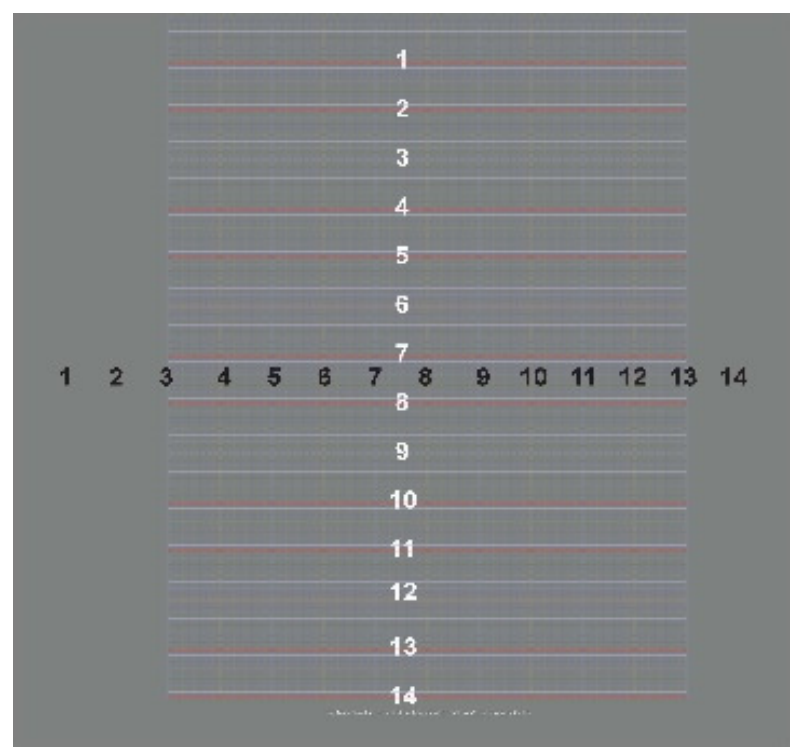

Figure 2: First Attempt with Centered Grid

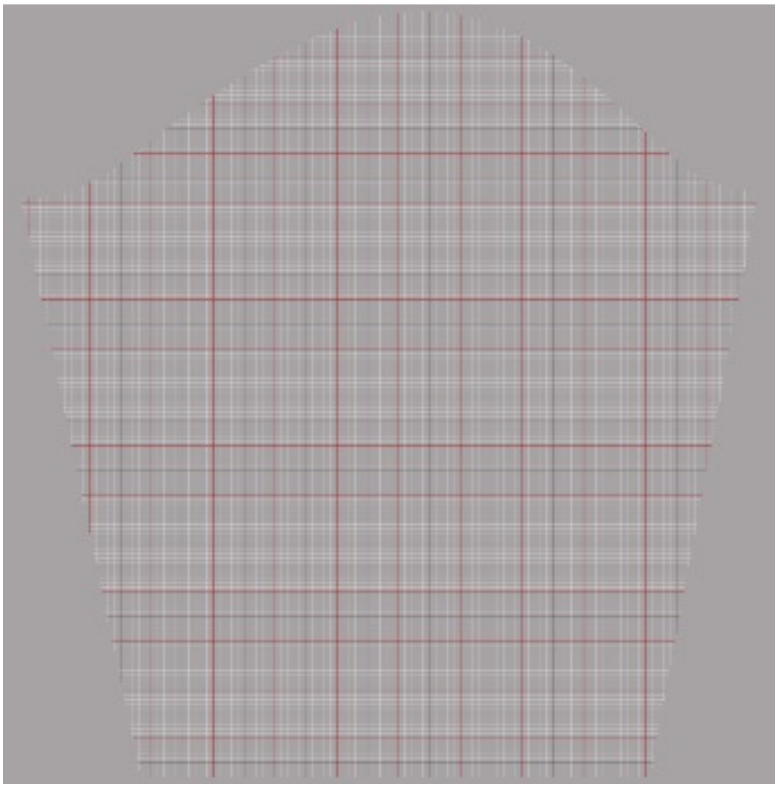

Figure 3: Second Attempt- Sleeve Pattern 
Technical designers spend endless hours measuring sample garments and comparing these garments to their standard. The goal of the fit fabric is to improve fit, lessen the rounds of sampling, and improve the overall ease of work for a technical designer. This method will specifically help in measuring and fitting first round samples and pattern draping. The printed squares on the finished garment will give the designers visual clues to determine whether the fabric is on grain, whether the measurements are correct, as well as if it is fitting properly (printed grid would be warped if it was? not fitting properly). If an adjustment needs to be performed, the grid acts as a measuring device to ensure that the exact adjustment can take place.

Once the pattern pieces for a particular garment have been developed in a CAD system, a grid structure pattern can be printed within the edges of the pattern even applying the exact amount of seam allowance without the grid and visual notches. This will provide the sample maker with more knowledge of how the garment will be constructed. A variety of fabrics with different characteristics such as weave, weight and drapability could be printed on demand as soon as the patternmaker's pattern is completed.

Fit fabric has the potential to replace muslin creating a whole line of 'better fabrics' for draping and fitting purposes. According to Gina Pisut and Lenda Connell of Auburn University, female fit preferences are very exact and over $80 \%$ of women can find at least two areas of their body where garments do not regularly fit them (2007). Use of the fit fabric could greatly improve this percentage by identifying those problem areas and correcting fit issues for the target customer. Some common forms of making adjustments are cutting open and pinning the sample, drawing in the lines where the sample should hit, taking pictures of manual adjustments and drawing the desired updates in a computer aided design program. These drawings and pictures are then either updated by a patternmaker or sent to the manufacturer to be updated in the pattern. This can lead to miscommunication. With this fit fabric, which has lines marked down to $1 / 8$ ", it will be much easier to communicate to the team how the adjustment is to be made. Another improvement for the designer is the ease of drawing lines and cutting the sample since the measurements are so clearly marked.

\subsection{Size Stream 3D Body Scanner}

This new 'fit fabric' has the potential then to utilize new technologies, such as 3D body scanning, or virtual fitting. There is an opportunity for the fit fabric system to be incorporated with 3D body scanning. The Size Stream 3D body scanner has the ability to produce a single color image in 3D body scanning, and now with its most recent updated software 14 high quality images are available; one from each sensor position. Combine this with accurate measurement data from the body scanner, this provides a method to compare actual flat measurements with on the body measurements.

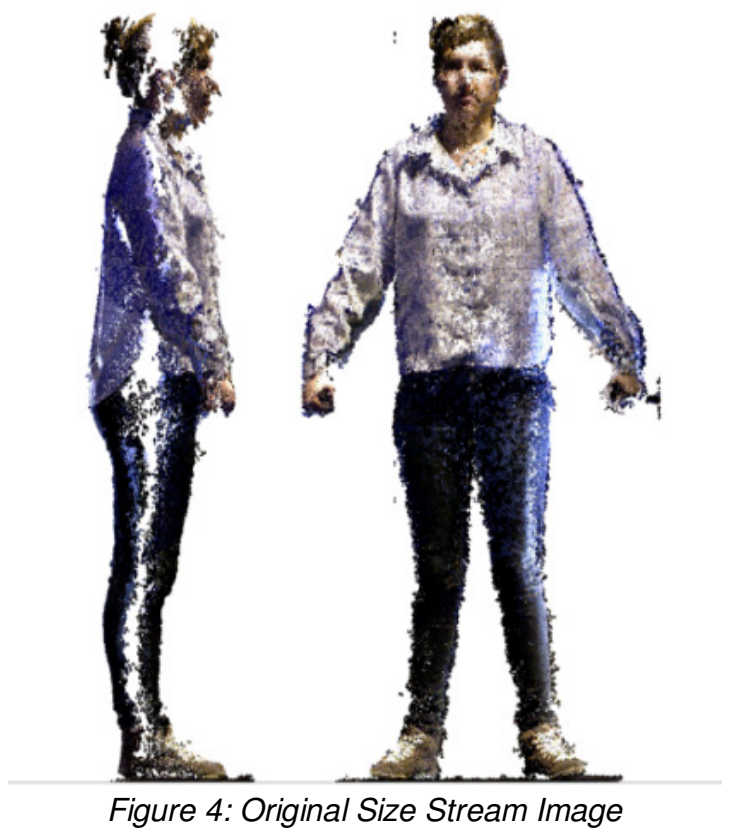




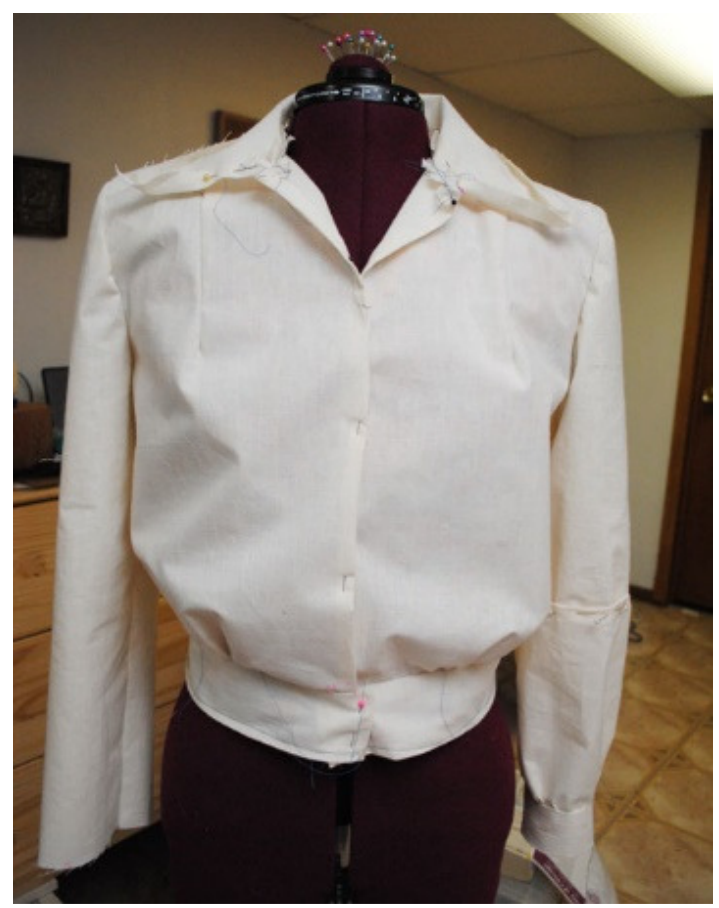

Figure 5: Typical Muslin Garment

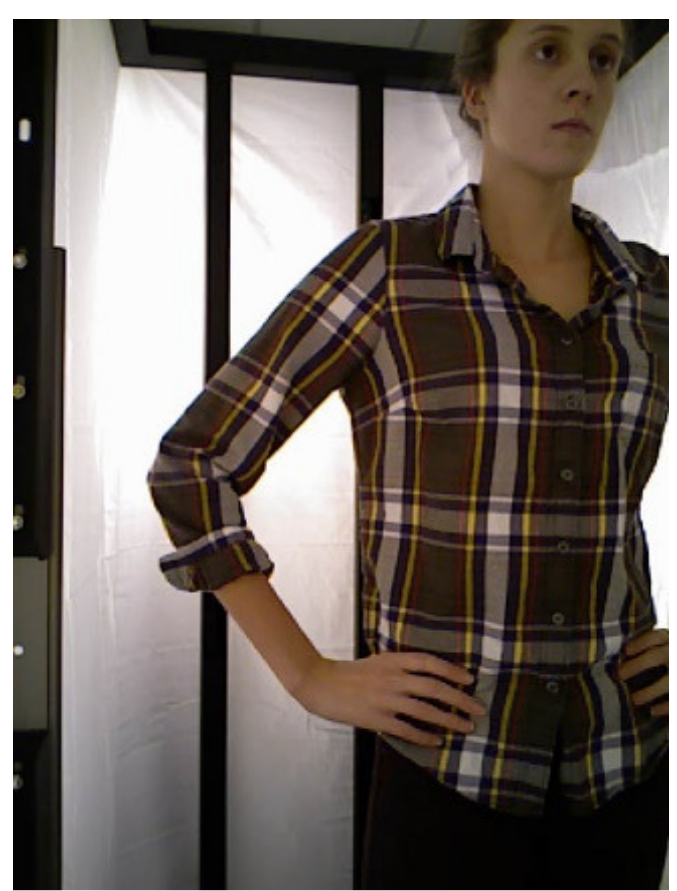

Figure 6: 1 of 14 high quality photos available at the time the body scan is taken

Testing can be performed through two methods: human testing where designers use the product and give feedback, and testing using color scanning from a 3D body scanner. The color scan technology combined with measurement extraction technology will allow measurement lines to appear on the color body scan, which will show the image of the person wearing the fit fabric garment. If the garment fits properly, the measurement lines on the garment made of fit fabric will match up with the measurement extraction lines appearing on the software. 


\section{Process and Methods}

Today, most consumer purchases are returned to the store due to poor fit (Anderson, 2004). This fabric system can expedite and improve the fit process by making pattern and measurement corrections, and reducing the amount of sample iterations. One drawback of using this fabric as compared to using muslin is cost. This fabric will be more expensive than muslin, however, as labor costs increase, especially for skilled patternmakers, technical designers and designers; the fabric costs become less of a factor. The fit fabric will make up for the added cost with fit accuracy and decreased product development time. This fit fabric will also likely be advantageous in markets where fit is emphasized, for example men's suits and women's pants.

\section{Analysis and Conclusions}

The concept of the fit fabric was originally conceived through an experiment with color images in 3D body scanning. The focus of the fit fabric is to assist technical designers, sample makers and pattern makers. As applications for this technology were examined, the concept of the fit fabric was conceived because the researchers identified that fit could be evaluated through the combination of color and 3D body scans. Much time is wasted in the apparel industry measuring sample garments and evaluating multiple rounds of samples. With the fit fabric, much of this currently wasted time will be reclaimed. In addition, measurements will be more accurate and therefore common sample issues will be evident.

\section{References}

Anderson, G. (2004). If the clothes fit, buy 'em. RetailWire. Retrieved from http://www.retailwire.com/discussion/10058/if-the-clothes-fit-buy-em

Brown, P. \& Rice, J. (2001). Ready-to-wear apparel analysis. Upper Saddle River, New Jersey: Prentice Hall.

Joseph-Armstrong, H. (2008). Draping for apparel design. New York, New York: Fairchild Publications. 